American Journal of Applied Sciences 7 (5): 661-668, 2010

ISSN 1546-9239

(C) 2010Science Publications

\title{
The Implications of a Health Promotion Program on the Knowledge and Practice of Automotive Workers Exposed To Solvent
}

\author{
${ }^{1}$ Rumaizah Ruslan, ${ }^{1}$ Zailina Hashim, ${ }^{2}$ Ismi Arif Ismail, ${ }^{3}$ Lin Naing and ${ }^{4}$ Rusli Nordin \\ ${ }^{1}$ Department of Community Health, Faculty of Medicine and Health Sciences, \\ ${ }^{2}$ Department of Professional Developments and Continuing Education, \\ Faculty of Educational Studies, University Putra Malaysia, 43400 Serdang, Selangor, Malaysia \\ ${ }^{3}$ Department of Community Health, Institute of Medicine, University Brunei Darussalam, \\ Jalan Tungku Link, Gadong BE 1410, Brunei Darussalam \\ ${ }^{4}$ Department of Public Health, School of Medicine and Health Sciences, Monash University, \\ JKR 1235, Bukit Azah, 80100 Johor Bahru, Johor, Malaysia
}

\begin{abstract}
Problem statement: An in-house health promotion program aimed at promoting safety and health awareness when handling organic solvents at the workplace was carried out and its effectiveness was evaluated by comparing the Knowledge, Attitude and Practices (KAP) scores at the pre and postintervention phase. Approach: A total of 104 workers participated in the program and another 176 workers served as the comparative group. Questionnaire was distributed to respondents for the program evaluation on the KAP as well as to obtain their background information. The program consisted of small group lectures and discussions, a short video show, display of posters and distributions of pamphlets. Results: Pre-intervention data showed poor practice and knowledge scores on solvent hazards $(<80 \%)$ among all respondents. There was a significant difference in the scores of knowledge $(\mathrm{p}<0.001)$ and practice $(\mathrm{p}=0.04)$ between the pre- and post-intervention phase among the intervention group. The intervention group had significantly higher scores in knowledge than the comparison group at the post-intervention phase. Findings showed that practice was not significantly correlated with knowledge, however, it was influenced by section employment as reflected by the length of time they had been working in various sections of the Paint Department. Conclusion: There were improvements in the KAP scores of the intervention group at the post-intervention phase. However, the practice was not significantly correlated to the knowledge, instead, it was influenced by the employment years in the heavily solvent exposed sections in the Painting Department indicating that their job experience and peer group interactions contributed to their work culture and behavior.
\end{abstract}

Key words: Health promotion program, knowledge, attitude, practices, automotive

\section{INTRODUCTION}

Health promotion emphasized on gaining or regaining control over personal well-being and it encourages a healthy psychosocial environment. It not only encourage healthy work practices among individuals through awareness and training programs, but also to develop and maintain of good working condition conducive to the well-being of the workforce as a whole (Asogawa et al., 1993). Health promotion in other word is a comprehensive process of developing and providing planned learning experiences to provide information, to change attitudes and to influence behavior (Anspaugh et al., 1995). Workplace safety and health promotion plays an essential role in every industry as one of the preventive measure in maintaining healthy and productive workers. It was expected to increase both behavior and normative belief, thus enhancing cognition and modifying attitudes towards safety and health issues (Hong et al., 2004). In this study, the program was known as a 'Safety and Health Program towards Solvent'. It was an approach towards the safe measures of handling chemical hazards at the workplace. As there were fewer local studies that focused on the safety and health promotion program especially in industrial setting, this study would provide overview of knowledge, attitude and practices status among workers exposed to solvent.

Corresponding Author: Zailina Hashim, Department of Community Health, Faculty of Medicine and Health Sciences, University Putra Malaysia, 43400 Serdang, Selangor, Malaysia Tel: 603-8947 2406 
The main module was on the knowledge of safety and health, especially to the workers who were using solvent in their daily job tasks. Risk communication was carried out by conveying the information to workers on the workplace hazards. This could raise the level of their awareness, minimize the risk of adverse health effects and encourage readiness to the workers on certain safety behaviors. Lower risk can be expected when workers recognized the hazards better and carry out safe work practice. This study was aimed at comparing the Knowledge, Attitude and Practices (KAP) score of 2 with knowledge and attitude score were determined. Other confounding factors that might influence the scores such as age, education years, employment years and period of employment in the sections of the Painting Department were also identified.

\section{MATERIALS AND METHODS}

Location and work sections: The study was conducted in an automotive industry in Malaysia. Toluene and xylene are the most common organic solvents use in the Painting Department of this industry studied. The 4 sections in the department where solvents were mainly used are the Mixing Room where chemical substances are mixed together to prepare for the specific paint according to the color code, the Primer Booth is where the car bodies are prepared before the second layer coating and spraying process on the inner part of the body which is carried out manually. The Top Coat Body Preparation and the Top Coat Spray Booth are where the car bodies are prepared for the third layer of coating and the spraying process on the inner part of the body which is also carried out manually.

Participants: A total of 280 male workers involved in this quasi-experimental study. They were exposed to organic solvents especially toluene and xylene, which were mainly used in thinners. A hundred and four workers volunteered to participate in the program as they have more time to spare. Those who did not volunteer were classified as the comparison group $(\mathrm{n}=176)$.

The safety and health program: The program was carried out in the training room at the Paint Department. The intervention group was divided into small groups which consisted of 15 participants. It was carried out by disseminating information through small group lectures and discussions, an audio-visual session and printed materials distribution (posters and pamphlet). The questions covered in the validated KAP questionnaire (Naing et al., 2006) was based on the information provided by the short lectures, discussions and printed materials, held in 2 phases. For the 1st phase, an interactive lecture, poster and pamphlet were distributed with Questions and Answer (Q and A) sessions carried out. After a $10 \mathrm{~min}$ break, the 2nd phase of the program was carried out which consisted of an audio-visual session and a short Q and A session on the potential risk, hazard and accidents that might occur in their groups (intervention and comparison group) at two phases in which evaluation were made on the KAP scores at the pre and post intervention phase (after 2 weeks). The correlations of practices workplace. After the time period of 2 weeks, participants were traced back and requested to fill in the same KAP questionnaire again.

The program content: The program consisted of information on the hazards of solvents to the respiratory, nervous system and skin, the sign and symptoms, the treatment, safe handling of solvents, health seeking behavior, control of hazards and general safe practice including the use of Personal Protective Equipment (PPE).

The program evaluation: The validated KAP questionnaire (Naing et al., 2006) was distributed to respondents 3 times at the 'pre-intervention', 'postintervention' and ' 3 months follow-up' after the post intervention. This paper only discuss on the pre and post-intervention phase. The purpose of the preintervention was to establish a baseline data on the workers' KAP. The difference of the KAP scores at pre and post-intervention phase indicated the effectiveness of the program.

Calculation of KAP scores: All questions covered various aspects of KAP. For knowledge on solvent as hazard and knowledge on control of hazard, there were 3 questions for each with answers such as "Yes", "Don't Know" and "No". For attitude, there were 5 questions with the responses such as "Strongly Do Not Agree", "Do Not Agree", "Neutral”, "Agree” and "Strongly Agree". The response for 10 of the practice questions were "Always", "Frequently", "Seldom" and "Never".

The KAP questionnaire comprised positive and negative questions. The positive questions were those with positive answers, in which "Yes" was the correct 
answer. If a question had a correct answer, then the score would be the highest. For example, in the Knowledge Section, the highest score would be 2 . However, if the answer is "No" or "Don't know", the score given would be 0 or 1 respectively. Negative question is with "No" as the correct answer, whereby, the score given would be the highest score of 2 marks. However, if the answer is "Yes" or "Don't know", the score would be 0 or 1 respectively.

According to the scoring system, the standard KAP score is in a form of percentage. The level below $80 \%$ of the total score in the Knowledge and Practices Sections and below $70 \%$ of the total score in the Attitude Sections indicated poor performance and considered as "need improvement" or "inadequate".

\section{RESULTS}

Background information: Out of 280 respondents were involved in the pre-intervention interview, only 104 volunteered to be in the intervention group. Meanwhile, 176 workers who did not volunteer which gave the response rate of $59.1 \%$, were put as the comparison group. Socio-demographic data were collected through questionnaire interview. All respondents involved were male workers, as the automotive manufacturing industry is a male-dominated industry. With reference to Table 1 , there was no significant difference in the mean age between the groups. The mean age was 26 years old and the majority had a mean of 12.5 years of formal education. Their mean employment was 6 years and worked 6 days per week, from 9-10 h daily including break time. Table 1 shows that the two groups were matched in term of their background.

Work flow and job process: The work sections where the respondents were selected were from the early process of non-painted car, known as white-body until the end of the process of painted body. The respondents were from all these sections. Table 2 shows the job descriptions of the respondents.

Knowledge, Attitude and Practices (KAP) scores of respondents: From the results, the respondents did not use goggle, did not undergo any training in chemical handling and were not provided with shower to clean up after work. The respondents were not aware or had poor knowledge on the hazards of solvent to the nervous system especially with late treatment. Low understanding and poor practices were indicated by the low percentage the score (below 80\%) for the positive questions (Table 3).

Table 1: Background information of respondents

\begin{tabular}{|c|c|c|c|c|c|c|}
\hline \multirow[b]{2}{*}{ Variables } & \multicolumn{2}{|c|}{ Intervention group $(\mathrm{n}=104)$} & \multicolumn{3}{|c|}{ Comparison group $(n=176)$} & \multirow[b]{2}{*}{$\mathrm{p}^{\mathrm{a}}$} \\
\hline & Mean (SD) & Range & Mean (SD) & Range & $t^{a}$ & \\
\hline \multicolumn{7}{|l|}{ Socio-demographic information } \\
\hline Age (year) & $26.26(2.93)$ & $20-34$ & $26.36(4.40)$ & $20-41$ & -0.216 & 0.830 \\
\hline Education (year) & $12.50(1.26)$ & $7-17$ & $12.30(0.99)$ & $9-15$ & 1.536 & 0.126 \\
\hline \multicolumn{7}{|l|}{ Work profile } \\
\hline Employment (year) & $5.76(3.67)$ & $1-30$ & $5.89(3.45)$ & $1-16$ & -0.237 & 0.813 \\
\hline Employment in the section (year) & $4.95(2.69)$ & $1-14$ & $5.08(3.36)$ & $1-15$ & -0.330 & 0.742 \\
\hline Work days (per week) & $5.51(0.75)$ & $5-7$ & $5.59(0.82)$ & $5-7$ & -0.731 & 0.465 \\
\hline Work hours(per day) & $9.63(1.38)$ & $8-13$ & $9.27(1.30)$ & $8-12$ & 0.350 & 0.211 \\
\hline Overtime hours (per week) & $19.73(6.55)$ & $2-26$ & $18.97(6.98)$ & $2-32$ & 0.900 & 0.369 \\
\hline
\end{tabular}

${ }^{\mathrm{a}}$ Independent t-test

Table 2: Work sections and the job descriptions in the Paint Department

\begin{tabular}{ll}
\hline Work section & Job tasks \\
\hline Plastisol Vinyl Chloride (PVC) & Sealing the bottom of the body and spraying the tire housing by using sealer. \\
Dry sanding & Clean up dusts on the body before entering the next section process. \\
Sealer and asphalt sheet & Installation of asphalt sheet at the bottom part of the body, adding sealer at each door. \\
Primer body preparation & Preparation before second paint coating. \\
Primer spray booth & Manual painting that is done by spraying paint on the inner part of the body. \\
& Major painting is done by the paint robot. \\
Wet sanding & Repairing the primer spray from any defect. \\
Top coat body preparation & $\begin{array}{l}\text { Preparing for the third layer of paint. } \\
\text { Top coat spray booth }\end{array}$ \\
& $\begin{array}{l}\text { Manual painting of the third layer of paint on the inner part of the body. } \\
\text { The process is done by spraying method. Major painting is done by the paint robot. }\end{array}$ \\
Online and offline repair & Repairing any minor defect from the painting process on and off the production line. \\
Wax booth & Applying wax on the painted body. \\
Mixing room & Preparing paints and solvents according to requested color coding.
\end{tabular}


Am. J. Applied Sci., 7 (5): 661-668, 2010

Table 3: Pre-intervention scores weak areas for both groups

\begin{tabular}{|c|c|c|}
\hline \multirow[b]{2}{*}{ Items } & \multicolumn{2}{|l|}{ Right answer (\%) } \\
\hline & Intervention group $(n=104)$ & Comparison group $(\mathrm{n}=176)$ \\
\hline \multicolumn{3}{|l|}{ Knowledge of solvent hazards on } \\
\hline Nervous system: & 24.04 & 28.44 \\
\hline Exposure to organic solvents can cause irritation & 23.08 & 26.14 \\
\hline \multicolumn{3}{|l|}{ Exposure to organic solvents can cause depression } \\
\hline \multirow{2}{*}{$\begin{array}{l}\text { Late treatment is easily available for the serious nervous } \\
\text { system illnesses due to solvent fumes }\end{array}$} & 20.19 & 22.16 \\
\hline & \multicolumn{2}{|l|}{ Right practice (\%) } \\
\hline \multicolumn{3}{|l|}{ General practice } \\
\hline Wear goggles while working & 11.54 & 9.66 \\
\hline Shower immediately after handling chemicals & 10.58 & 17.61 \\
\hline Undergo training in chemicals use & 12.50 & 14.20 \\
\hline
\end{tabular}

Table 4: Comparison of KAP during the pre and post intervention phases between the 2 groups

\begin{tabular}{|c|c|c|c|c|c|c|c|c|}
\hline & \multicolumn{4}{|c|}{ Pre-intervention mean (SD) } & \multicolumn{4}{|c|}{ Post-intervention mean (SD) } \\
\hline & \multirow{2}{*}{$\begin{array}{l}\text { Intervention group } \\
(\mathrm{n}=104)\end{array}$} & \multicolumn{3}{|c|}{ Comparison group } & \multirow{2}{*}{$\begin{array}{l}\text { Intervention group } \\
(\mathrm{n}=104)\end{array}$} & \multicolumn{3}{|c|}{ Comparison group } \\
\hline & & $(\mathrm{n}=176)$ & $\mathrm{t}$ & $\mathrm{p}$ & & $(n=176)$ & $\mathrm{t}$ & $\mathrm{p}$ \\
\hline \multicolumn{9}{|c|}{ Knowledge (solvent as hazard) } \\
\hline Respiratory & $87.2(8.3)$ & $86.0(9.8)$ & 0.993 & 0.322 & $92.4(6.7)$ & $88.0(10.2)$ & 4.360 & $0.001 * * *$ \\
\hline Nervous system & $76.6(11.2)$ & $76.0(12.5)$ & 0.592 & 0.692 & $88.5(9.8)$ & $79.0(14.3)$ & 6.538 & $0.001 * * *$ \\
\hline Skin & $84.3(13.0)$ & 84.9 (13.5) & -0.375 & 0.708 & $92.1(9.7)$ & $87.1(14.0)$ & 3.543 & $0.001 * * *$ \\
\hline \multicolumn{9}{|c|}{ Knowledge (control of hazard) } \\
\hline Respiratory & $90.8(8.4)$ & $91.7(8.0)$ & -0.872 & 0.384 & $94.6(6.7)$ & $92.0(8.5)$ & 2.784 & $0.006 * *$ \\
\hline Nervous system & $91.0(8.4)$ & $91.4(7.2)$ & -0.402 & 0.688 & $95.4(6.1)$ & $91.9(8.4)$ & 4.113 & $0.001^{* * *}$ \\
\hline Skin & $90.7(9.0)$ & $91.2(7.7)$ & -0.419 & 0.675 & $94.5(6.8)$ & 91.2 (8.9) & 3.439 & $0.001^{* * *}$ \\
\hline \multicolumn{9}{|l|}{ Attitude } \\
\hline Respiratory & $81.8(10.5)$ & $83.8(10.7)$ & -1.473 & 0.142 & $82.1(11.4)$ & $81.6(12.7)$ & 0.334 & 0.739 \\
\hline Nervous system & $81.8(10.8)$ & $83.6(10.5)$ & -1.395 & 0.164 & $82.4(11.5)$ & $81.8(12.5)$ & 0.404 & 0.686 \\
\hline Skin & $81.3(10.6)$ & $82.8(10.7)$ & -1.148 & 0.252 & 82.0 (11.6) & $81.0(12.7)$ & 0.656 & 0.512 \\
\hline Practices & $72.6(13.7)$ & $72.2(15.1)$ & 0.251 & 0.802 & 75.6 (15.3) & $73.3(17.2)$ & 1.107 & 0.269 \\
\hline
\end{tabular}

***: Significant at $\mathrm{p}<0.001$

Table 5: Comparison of KAP scores between the pre and post intervention phase among the intervention group

\begin{tabular}{|c|c|c|c|c|}
\hline \multirow[b]{2}{*}{ Items } & \multicolumn{4}{|l|}{ Mean (SD) } \\
\hline & Pre-intervention & Post-intervention & $t^{\mathrm{a}}$ & $\mathrm{p}^{\mathrm{a}}$ \\
\hline \multicolumn{5}{|c|}{ Knowledge (solvent as hazard) } \\
\hline Respiratory & 87.2 (8.3) & $92.4(6.7)$ & -5.402 & $0.001 * * *$ \\
\hline Nervous system & $76.6(11.2)$ & $88.5(9.8)$ & -9.744 & $0.001^{* * *}$ \\
\hline Skin & $84.3(13.0)$ & $92.1(9.7)$ & -5.454 & $0.001^{* * *}$ \\
\hline \multicolumn{5}{|c|}{ Knowledge (control of) } \\
\hline Respiratory & $90.8(8.4)$ & $94.6(6.7)$ & -4.502 & $0.001 * * *$ \\
\hline Nervous system & $91.0(8.4)$ & $95.4(6.1)$ & -5.005 & $0.001^{* * *}$ \\
\hline Skin & $90.7(9.0)$ & $94.5(6.8)$ & -4.06 & $0.001 * * *$ \\
\hline \multicolumn{5}{|l|}{ Attitude } \\
\hline Respiratory & $81.8(10.5)$ & $82.1(11.4)$ & -0.237 & 0.813 \\
\hline Nervous system & $81.8(10.8)$ & $82.4(11.5)$ & -0.431 & 0.667 \\
\hline Skin & $81.3(10.6)$ & $82.0(11.6)$ & -0.580 & 0.563 \\
\hline Practices & $72.6(13.7)$ & 75.6 (15.3) & -2.067 & $0.041 *$ \\
\hline
\end{tabular}

$\mathrm{n}=104 ;^{\mathrm{a}}$ : Paired t-Test; *: Significant at $\mathrm{p}<0.05 ;{ }^{* * *}$ : Significant at $\mathrm{p}<0.0$

Comparison of KAP scores between the two groups during the pre and post-intervention program: The two groups' KAP scores from the pre and the postintervention phases were compared. No significant difference in the scores between both groups was found before the intervention program. However, there were significant differences in scores for both groups on the "knowledge of solvent as hazards" and "knowledge on the control of hazard" at the post intervention phase, whereby, the intervention group had significantly higher scores than the comparison group (Table 4).

Comparison of KAP scores for the pre and postintervention program among the intervention group: The results indicated that there were significant differences for the scores of knowledge of solvent as hazard and knowledge on the control of hazard and practices between the pre and post intervention phases. 
The mean scores of KAP for the intervention group in the three domains of respiratory, nervous system and skin (RNS) were significantly higher at the post intervention phase (Table 5). However, no significant difference in the attitude scores between the pre and post- intervention found.

Comparison of KAP scores during pre and postintervention program among the comparison group: The score of knowledge on solvent as hazard in RNS domains among comparison group had increased after the awareness program. Contrary to this, the knowledge on the control of hazards and practice scores were not significantly higher than the pre-intervention phase
(Table 6). However, the attitude scores of the post intervention phase were higher than the pre-intervention phase.

Factors which influenced the KAP scores: There was no significant relation between practice with knowledge as well as with attitude scores (Table 7). Other variables such as age, formal education levels, employment years and the employment in the Painting Department, which might influence the KAP scores, were controlled. For all the KAP scores, only the employment in the Paint Department showed a significant influence for almost $7.5 \%$ of the variance in the practices score (Table 8 ).

Table 6: Comparison of KAP scores between the pre and post intervention phase among the comparison group

\begin{tabular}{|c|c|c|c|c|}
\hline \multirow[b]{2}{*}{ Items } & \multicolumn{4}{|l|}{ Mean (SD) } \\
\hline & Pre-intervention & Post-intervention & $\mathrm{t}^{\mathrm{a}}$ & $\mathrm{p}^{\mathrm{a}}$ \\
\hline \multicolumn{5}{|c|}{ Knowledge (solvent as hazard) } \\
\hline Respiratory & $86.0(9.8)$ & $88.0(10.2)$ & -3.334 & $0.001 * * *$ \\
\hline Nervous system & $76.0(12.5)$ & $79.0(14.3)$ & -3.880 & $0.001 * * *$ \\
\hline Skin & $84.9(13.5)$ & $87.1(14.0)$ & -3.258 & $0.001 * * *$ \\
\hline \multicolumn{5}{|c|}{ Knowledge (control of hazard) } \\
\hline Respiratory & $91.7(8.0)$ & $92.0(8.5)$ & -0.676 & 0.500 \\
\hline Nervous system & $91.4(7.2)$ & $91.9(8.4)$ & -0.857 & 0.393 \\
\hline Skin & $91.2(7.7)$ & $91.2(8.9)$ & -0.105 & 0.916 \\
\hline \multicolumn{5}{|l|}{ Attitude } \\
\hline Respiratory & $83.8(10.7)$ & $81.6(12.7)$ & 2.463 & $0.015^{*}$ \\
\hline Nervous system & $83.6(10.5)$ & $81.8(12.5)$ & 2.091 & $0.038^{*}$ \\
\hline Skin & $82.8(10.7)$ & $81.0(12.7)$ & 2.001 & $0.047^{*}$ \\
\hline Practices & $72.2(15.1)$ & $73.3(17.2)$ & -1.030 & 0.304 \\
\hline
\end{tabular}

Table 7: Correlation of practices with knowledge and attitude scores based on the 3 domains-respiratory, nervous system and skin (RNS)

\begin{tabular}{lccc}
\hline Practices & Mean (SD) & $\mathrm{r}^{\mathrm{a}}$ & $\mathrm{p}^{\mathrm{a}}$ \\
\hline Knowledge (solvent as hazard) & & & 0.001 \\
Respiratory & $92.36(6.68)$ & 0.014 & 0.228 \\
Nervous system & $88.46(9.30)$ & 0.011 & 0.279 \\
Skin & $92.12(9.67)$ & & 0.012 \\
Knowledge (control hazard) & & 0.009 & 0.261 \\
Respiratory & $94.57(6.69)$ & 0.028 & 0.340 \\
Nervous system & $95.43(6.12)$ & & 0.087 \\
Skin & $94.47(6.79)$ & 0.032 & 0.070 \\
Attitude & & 0.032 & 0.067 \\
Respiratory & $82.12(11.38)$ & 0.027 & 0.095 \\
Nervous system & $82.36(11.51)$ & \\
Skin & $82.02(11.56)$ & & \\
\hline$:$ Peason
\end{tabular}

${ }^{\mathrm{a}}$ : Pearson correlation

Table 8: Variables that significantly influenced the practices among the intervention group

\begin{tabular}{llrl}
\hline Selected variables & $\mathrm{b}^{\mathrm{a}}(95 \% \mathrm{CI})$ & $\mathrm{t}$ & $\mathrm{p}$ \\
\hline Age (year) & $-0.957(-2.391,3.670)$ & 0.323 & 0.553 \\
Education (year) & $0.644(-1.667,0.517)$ & -1.074 & 0.581 \\
Employment (year) & $0.610(-1.737,2.956)$ & 2.713 & 0.285 \\
Employment in Paint Department (year) & $2.120(0.569,0.478)$ & 0.008 \\
\hline
\end{tabular}

$\mathrm{n}=104 ; \mathrm{R}^{2}=0.075 ;{ }^{\mathrm{a}}$ : Multiple linear regression (ENTER method) 
Am. J. Applied Sci., 7 (5): 661-668, 2010

\section{DISCUSSION}

Only 104 out of 280 workers were involved in the intervention program, which gave a response rate of $37 \%$. There was a high production request at the time when the research was conducted, which increased the workload and commitment of workers from all sections. Based on the 2008 news market reports, the manufacturing rate was increased to 200,000 units in 2008, indicating the high production level (BERNAMA, 2008). The year sales had reached 167,000 units, making a great achievement for the local car maker industry compared to the 2007 sales with only 166,700 units sold (BERNAMA, 2009; 2010). Due to the demands, the period for the intervention program was limited to one and a half hours daily and the participation from the respondents was optimized.

There were 13 major sections involved in this study as shown in Table 2. The job tasks in these sections involved the use of solvents especially in paint and thinners. There were also others chemicals used such as sealer or wax; however, solvents are the major chemical used.

Based on the knowledge scores, the score of below $80 \%$ indicated inadequate knowledge whereby, there was a need for improvement on the level of their understanding. The results showed that "knowledge of solvents as hazard" on the nervous system were below the standard score (Table 4). The knowledge score was narrowed to each question from the KAP questionnaire in order to identify the weak areas. The questions included were "exposure to organic solvents can cause irritation", "exposure to organic solvents can cause depression" and "late treatment is easily available for the serious nervous system illness due to solvent inhalation" (Table 3). These subjects were highlighted in the health promotion program, which were later continued along with the issues of the signs and symptoms, prognosis of the illness, treatment, safety behavior, Personal Protective Equipment (PPE) and good work practices. The content of the intervention program should be according to the workers' need. Koh (1995) suggested that preventive strategies should direct towards environmental control as well as the enhancement of lifestyle behaviors. By comparing work-related illness and general illness, it would be useful for the promotion of positive health. It would be appropriate to link up the general illnesses with fitness to work and common causes of morbidity such as smoking. This is because the impact of morbidity due to general illness and unhealthy lifestyles may overweight the morbidity due to occupational ill health (Koh, 1995).
The practices scores of below $70 \%$ (Table 4), indicated poor and inadequate work practices. The questions focused were "do you wear goggle while working”, "do you shower immediately after working with chemicals" and "do you attend training program in chemical use" (Table 3). Based on the responses to questions in the interview sessions using the questionnaire, respondents did not use the goggles provided, because of poor visibility due to the effect of residual spray paint left on the goggle. Instead, they preferred spraying without wearing goggles. No shower facilities were provided in the workplace for the workers to clean themselves after work, thus they could not practice proper hygiene and responded negatively to the questions.

There was a lack of training on safe chemicals use, which resulted in poor practices among the workers. Not all respondents had the opportunity to be involved in focused training. Besides, the training that had been held previously was basically more on safety rather than on the health issues. Even though the company had planned training programs on chemical handling, but they were not implemented yet at the time when this study was conducted. The training sessions were carried out by the company's own experts or other consultants from companies, beside personnel from the Department Of Safety and Health (DOSH) and the National Institute of Occupational Safety and Health (NIOSH). By the year 2007, there were 1036 health and safety promotional activities implemented by DOSH. There were Safety and Health Promotions Week, Safety and Health Award, seminars and dialogue sessions held by the company. Meanwhile, NIOSH had carried out 6716 series of OSH training with 159,000 participants during 2004 until 2007. The target groups were employees, OSH professional and managers (Zabidi et al., 2007).

The comparison between the intervention and comparison group were carried out during the pre and post-intervention program. No significant difference in the KAP scores between the two groups before the program was carried out. These were probably due to the "Safety Induction Training", a compulsory program provided for all employees at the early stage of their recruitment period.

At the post intervention phase, there was a significant improvement in "knowledge on solvent as hazard" and "knowledge on the control of hazard" as they relate to the health effects on the respiratory, nervous and skin systems among the intervention group. These findings indicated the effectiveness of the intervention program to enrich the respondents' awareness toward safety and health issues. The improved scores on knowledge after the intervention 
suggested that the workers were aware of the hazard and safety measures of solvent handling. From the scores, the basic information on solvents hazards, the health effects and preventive measures were well understood. A study carried out in a petrochemical industry documented that workers' safety and health training was related to hazard recognition (Hong et al., 2004). Increases in knowledge after training had been documented in the previous studies (Uskun et al., 2008; Harrington and Walker, 2004), however in different study conducted, the improvement in knowledge does not necessarily reflect on the reduced injury rates (Daltroy et al., 1997).

The comparison between KAP scores during the pre and post intervention for both groups showed the effectiveness of the program. For the intervention group, the significant difference in knowledge as well as practices' scores at the pre and post intervention phase means that the program had conveyed the information effectively to the respondents.

As for the comparison group, the better scores on "knowledge on solvent as hazard" were likely the results of peer group influence. The respondents from the intervention group might have shared their knowledge from the intervention program to those in the comparison group. Pamphlet, which was distributed to the participants and the posters displayed, might have also provided some safety and health information to the comparison group who were in the same work areas and thus slightly improved on their knowledge. This was supported by a previous study who suggested that socialized people are to some degree, pressured to act or behave in accordance with the attitudes and values agreed upon by the social groups to which they belong. It means that persons working towards specific solution of problems and towards making specific changes maintained the status quo. Workers shared problems and solutions with others in their group (Elkind, 1993). Another study stated that the work group is likely to function as a powerful modeling of "association network" for individuals because peer provides a primary source of social support and solidarity in the workplace (Olson et al., 2009). In addition, socialization into the workplace had affected on an individual's safety attitude and behavior especially among new employees who did not have experience before (Mullen, 2004).

Yet the information they gained were limited to their own perception without further explanation as to what they would get by attending the health promotion program. This was indicated by the attitude scores among the comparison during post-intervention time phases which were significantly lower than pre- intervention (Table 5). Even though the results showed in the comparison group had significantly increased in knowledge, the findings did not interfere with the evaluation of the program in which the intervention group significantly had better scores than the comparison after undergoing the health promotion program (Table 6).

Based on the findings, the practices were influenced by the employment years in the sections of the Painting Department. The findings suggested that the safe work practices or behavior were due to the work culture. In a study which found a definite workcultural trait in risk behavior associated with the workers' job. The manner in which they perceived hazards, the interactions with respect to fear and discourse while in the social settings all suggested risktaking and hazard-coping mechanism shared by the work culture (Elkind, 1993). This study had indicated that workers who have worked longer in these sections had practiced safety based on their daily job experiences. Inverse relationship between the unsafe work practice with age and work experience had been documented before (Nouri et al., 2008). The accident risk generally decreased as the work experience increased (Butani, 1988; Saari and Lahtela, 1981).

\section{CONCLUSION}

These findings showed significantly improved scores on knowledge and practices of the participants when compared to the comparative group. From the results, the health promotion program had improved the participants' knowledge on hazard and has promoted safe practices over a short time period after the implementation of the program. Findings showed that practice was not significantly correlated with knowledge, however, it was influenced by peer group and work experience as reflected by the length of time participants had been working in various sections of the Paint Department. It is recommended that routine health promotion program be implemented for the employees especially those who have more experience and worked longer so that they can be resource persons or role models for the new employees.

\section{ACKNOWLEDGEMENT}

This research was sponsored by the Research University Grant Scheme (RUGS) vote no: 91131. We would like to extend our thanks to Nur Farhana Mohd Nawal as the company's Health Safety Executive, Paint Department managerial staffs for supporting the study and also thanks to the respondents for completing the 
questionnaires and participating in the health promotion program.

\section{REFERENCES}

Anspaugh, D.J., S. Hunter and J. Mosley, 1995. The economic impact of corporate wellness programs: Past and future considerations. Am. Assoc. Occup. Health Nurse J., 43: 203-10. http://www.biomedsearch.com/nih/economicimpact-corporate-wellness-programs/7772205.html

Asogawa, S.E., S. Casswell, K. El Fawal, J. O’Connor and H. Sandoval-Ovellana et al., 1993. Report of a WHO expert committee on health promotion in the workplace: Alcohol and drug abuse. Geneva: World Health Organization. http://whqlibdoc.who.int/trs/WHO_TRS_833.pdf

BERNAMA, 2008. Perodua to maintain the current selling price. BERNAMA. http://web10.bernama.com/auto/newsDetail.php?id $=354570$

BERNAMA, 2009. Perodua expects 6-10 percent drop in sales. BERNAMA. http://web10.bernama.com/auto/newsDetail.php?id $=400301$

BERNAMA, 2010. Perodua eyes 176,000 New sales in 2010.

BERNAMA. http://web10.bernama.com/auto/newsDetail.php?id $=469825$

Butani, S.J., 1988. Relative risk analysis of injuries in coal mining by age and experience at present company. J. Occup. Accidents, 10: 209-216. DOI: 10.1016/0376-6349(88)90014-4

Daltroy, L.H., M.D. Iversen, M.G. Larson, R. Lew and E. Wright et al., 1997. A controlled trial of an educational program to prevent low back injuries. N. Eng. J. Med., 337: 322-328. http://content.nejm.org/cgi/reprint/337/5/322.pdf

Elkind, P.D., 1993. Correspondence between knowledge, attitudes and behavior in farm health and safety practices. J. Safe. Res., 24: 171-179. DOI: 10.1016/0022-4375(93)90028-L

Harrington, S.S. and B.L. Walker, 2004. The effect of ergonomics training on the knowledge, attitudes and practices of teleworkers. J. Safe. Res., 35: 13-22. DOI: 10.1016/J.JSR.2003.07.002
Hong, Y.J., Y.H. Lin, H.H. Pai, Y.C. Lai and N.I. Lee, 2004. Developing a safety and health training model for petrochemical workers. Kaohsiung J. Med. Sci., 20: 56-61. DOI: 10.1016/S1607551x(09)70085-3

Koh, D., 1995. Occupational health and safety promotion: Problems and solutions. Safe. Sci., 20: 323-328. DOI: 10.1016/0925-7535(95)00030$\mathrm{K}$

Mullen, J., 2004. Investigating factors that influence individual safety behavior at work. J. Safe. Res., 35: 275-285. DOI: 10.1016/J.JSR.2004.03.011

Naing, L., N. Rusli, M.Y. Khairol Reza, H. Zailina and H.H. Jamal et al., 2006. Validation of knowledge, attitude and practices questionnaire for solvent exposed workers: A pilot study. J. Commun. Health, Suppl., 1: 1-1.

Nouri, J., A. Azadeh and I. Mohammad Fam, 2008. The evaluation of safety behaviors in a gas treatment company in Iran . J. Loss Prev. Process Ind., 21: 319-325. DOI: 10.1016/J.JLP.2007.11.006

Olson, R., A. Grosshuesch, S. Schmidt, M. Gray and B. Wipfli, 2009. Observational learning and workplace safety: The effects of viewing the collective behavior of multiple social models on the use of personal protective equipment. J. Safe. Res., 40: 383-387. DOI: 10.1016/J.JSR.2009.07.004

Saari, J. and J. Lahtela, 1981. Work conditions and accidents in three industries. Scandinavian J. Work, Environ. Health, 7: 97-105. http://www.sjweh.fi/download.php?abstract_id=25 74\&file_nro=1

Uskun, E., S.B. Uskun, M. Uysalgenc and M. Yagiz, 2008. Effectiveness of a training intervention on immunization to increase knowledge of primary healthcare workers and vaccination coverage rates. Public Heath, 122: 949-958. DOI: 10.1016/J.PUHE.2007.10.005

Zabidi, M.A., A.R. Majahar, Y. Mohd Rosdee, S.A. Aziz and C.A. Husdin et al., 2007. Inculcating safety culture. Ministry of Human Resources. Putrajaya: Malaysia. http://www.dosh.gov.my/doshV2/phocadownload/ Publication/safety_culture.pdf 